\title{
$N$-溴代丁二酰亚胺催化水杨醛与麦氏酸反应合成香豆素-3-羧酸
}

\author{
阮鸿力 ${ }^{a}$ 张婧圆 ${ }^{a}$ 孙 赛 ${ }^{a}$ 杨 颖 ${ }^{a}$ 朱小硈 ${ }^{b}$ 吕成伟 $*, a$ \\ ( ${ }^{a}$ 辽宁师范大学化学化工学院 大连 116029) \\ ( ${ }^{b}$ 鞍山市教师进修学校 鞍山 14000)
}

\begin{abstract}
摘要 室温条件下、水和乙醇的混合液中, $N$-溴代丁二酰亚胺(NBS)促进 2-羟基苯甲醛与麦氏酸连续发生 Knoevenagel 缩合反应和分子内环化反应，高产率地合成了一系列的香豆素-3-羧酸. 该方法扩展了可用于合成香豆素-3-羧酸的催化 剂的种类，同时还具有催化剂廉价易得、反应条件清洁环保、底物适用性广、后处理简单和产物易于纯化等优点. 关键词 $N$-溴代丁二酰亚胺; 香豆素-3-羧酸; 合成方法; 室温; 绿色化学
\end{abstract}

\section{$N$-Bromosuccinimide Mediated the Reaction of 2-Hydroxyaryl Aldehydes with Meldrum's Acid for Synthesis of Coumarin-3-carboxylic Acids}

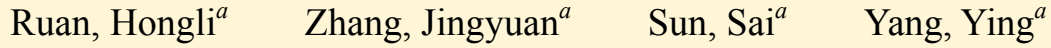 \\ Zhu, Xiaolei ${ }^{b} \quad$ Lü, Chengwei*,a \\ ( ${ }^{a}$ School of Chemistry and Chemical Engineering, Liaoning Normal University, Dalian 116029) \\ ( ${ }^{b}$ Teachers Training College of Anshan, Anshan 114000)
}

\begin{abstract}
A $N$-bromosuccinimide (NBS) promoted procedure for the synthesis of coumarin-3-carboxylic acids via Knoevenagel-intramolecular cyclization cascade reaction of Meldrum's acid with various 2-hydroxyarylaldehydes has been developed. Using the mixture of water and ethanol as solvent, employing inexpensive NBS as the catalyst, and reacted at room temperature are found to highly applicable to get a satisfactory outcome. This approach expands the method for the preparation of coumarin-3-carboxylic acids and also provides other features such as mild reaction conditions, tolerant the substrates with diverse functional groups, simple workup procedure and easy isolation.

Keywords $N$-bromosuccinimide; coumarin-33-carboxylic acids; synthetic methods; room temperature; green chemistry
\end{abstract}

自 “绿色化学” 的概念被提出以来, 溶剂的绿色化 和简单、高效、无害的催化剂的使用一直备受关注 ${ }^{[1,2]}$. 在有机反应过程中反应介质扮演着至关重要的角色, 溶 剂不仅能使催化剂与各组分反应物之间紧密接触, 还可 以在反应完成之后便于催化剂的回收和生成物的分离 纯化. 因此, 对于有机反应来说, 绿色、价廉、合适的溶 剂的选择成为了一个重要的研究课题 ${ }^{[3-5]}$. 近年来, 水 和乙醇的混合液作为溶剂被广泛的应用于合成各类重 要的有机化合物 ${ }^{[6 \sim 10]}$. 以水作溶剂、乙醇为辅助溶剂, 既具有水安全有效、价廉无毒、环境友好等优点, 又可 以克服单纯在水相中反应面临的对有机物溶解性差和
需要使用大量的有机溶剂萃取、经过繁复的后处理提纯 产物等问题 ${ }^{[11 ~ 13]}$. $N$-溴代丁二酰亚胺(NBS) 是一种非常 重要的有机物, 被广泛应用于有机合成反应中. 随着研 究的深入, NBS 的应用领域越来越多, 作为催化剂显示 出巨大的潜力及应用价值 ${ }^{[14 \sim 21]}$.

香豆素及其衍生物是一类广泛存在于自然界中的 杂环化合物, 在食品、医药、农药、香料、染料、光电 材料和超分子识别等领域都有重要的应用 ${ }^{[22 ~ 27]}$. 目前, 合成香豆素类化合物的方法主要包括 Perkin 反应、 Pechmann 反应、Knoevenagel 缩合反应、Wittig 反应、 Reformatsky 反应、Claisen 重排反应、Suzuki 偶联反应

* Corresponding author. E-mail: chengweilv@126.com
Received January 5, 2017; revised March 21, 2017; published online April 18, 2017.

Project supported by the National Natural Science Foundation of China (No. 21403100) and the Doctoral Scientific Research Foundation of Liaoning Province (No. 20141100).

国家自然科学基金(No. 21403100)和辽宁省博士启动基金(No. 20141100)资助项目. 
等 ${ }^{[24,28,29]}$. 其中利用水杨醛与含有活泼亚甲基的麦氏酸 发生 Knoevenagal 缩合-分子内环化反应是最简便的方 法之一, 可以有效地合成香豆素-3-羧酸类化合物 ${ }^{[30 ~ 32]}$. 对该方法的研究与改进已经成为国内外学者的热门研 究方向, 发展了多种酸性或碱性的催化剂促进该反应的 进行 ${ }^{[28,31,33 ~ 37]}$. 尤其是近两年, 在 $\mathrm{FeCl}_{3}{ }^{[38]} 、 \mathrm{Yb}(\mathrm{OTf})_{3}{ }^{[39]}$ 、 $\mathrm{NaN}_{3}{ }^{[40]}$ 、金合欢水萃物 ${ }^{[41]}$ 和柠檬汁 ${ }^{[42]}$ 的作用下, 在乙醇 或水相中反应实现了高产率的合成香豆素-3-羧酸. 但 该方法在进一步优化反应条件、简化操作过程、提高产 率和扩大底物适应范围等方面仍有很大的发展空 间 ${ }^{[40,42]}$. 我们课题组在这方面的研究取得了初步的进

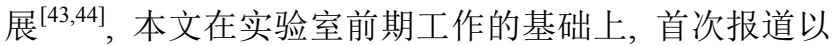
NBS 为催化剂, 水作为反应溶剂( 乙醇为辅助溶剂), 促 进水杨醛与麦氏酸在室温条件下发生反应合成香豆素3-羧酸(Eq. 1), 既安全环保, 又能够简化后处理和提纯 过程.

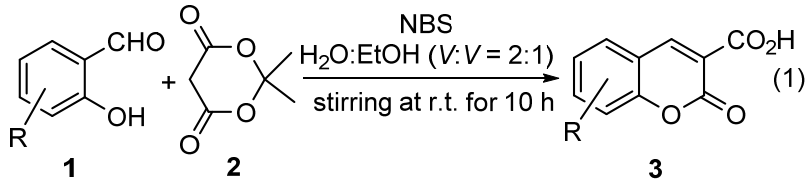

\section{1 结果与讨论}

\section{1 反应条件篮选}

为获取最佳反应效果, 我们以 $0.5 \mathrm{mmol}$ 水杨醛和 $0.5 \mathrm{mmol}$ 麦氏酸的反应为模型、NBS 为催化剂, 优化反 应条件, 结果见表 1. 因为水相中的反应添加乙醇作为
辅助溶剂可以克服使用纯水作为溶剂的缺陷，所以我们 使用水和乙醇的混合物作为溶剂，篮选二者的比例和用 量对反应的影响(表 1, Entries 1 6).

在水相中该反应只有 $70 \%$ 的收率，当水和乙醇的比 例为 $2: 1$, 溶剂总量为 $1.5 \mathrm{~mL}$ 时获得最佳产率(表 1 , Entry 5). 我们又考察了可以作为相转移催化剂的 PEG-400、与乙醇相似的甲醇和丙酮作为辅助溶剂的情 况(表 1, Entries 7～9). 虽然也可以提高反应的产率，但 水和乙醇的混合液作为溶剂效果最好. 研究催化剂的作 用时发现无催化剂的条件下只能获得 $80 \%$ 的收率，而使 用丁二酰亚胺作为催化剂的结果与不使用催化剂相当, 证明 $N$-溴代丁二酰亚胺确实能够促进反应进行(表 1 , Entries 10,11). 在考察物料比时发现增加麦氏酸的用量 到 $0.55 \mathrm{mmol}$, 产率提高到 94\%(表 1, Entry 12), 增大水 杨醛的用量产率没有变化(表 1, Entry13). 接下来我们对 催化剂的用量进行探究，当催化剂的用量降低到 15 $\mathrm{mol} \%$ 时, 产率略有提高(表 1, Entry 14), 继续减少催化 剂的用量则对反应不利(表 1, Entry 15). 最后，考察了反 应温度和时间对结果的影响(表 1, Entries 16 18). 如表 1 所示, 升高反应温度到 $40{ }^{\circ} \mathrm{C}$, 产率没有变化, 而降低 反应温度到室温，需要延长反应时间到 $10 \mathrm{~h}$ 可以达到同 样 $97 \%$ 的产率. 综上所述，我们选择以 $1.0 \mathrm{~mL}$ 水和 0.5 $\mathrm{mL}$ 乙醇为反应溶剂, $15 \mathrm{~mol} \%$ 的 NBS 为催化剂, 水杨醛 和麦氏酸以 $1: 1.1$ 的比例在室温条件下反应 $10 \mathrm{~h}$ 作为 最佳的反应条件.

在最佳反应条件下，我们系统的考察了不同取代的 水杨醛与麦氏酸的缩合反应，结果列于表 2 中. 可以看

表 1 反应条件篮选

Table 1 The screening of reaction conditions

\begin{tabular}{|c|c|c|c|c|c|c|}
\hline Entry $^{a}$ & Catalyst (mol\%) & Solvent & $V / \mathrm{mL}$ & Temp. $/{ }^{\circ} \mathrm{C}$ & Time/h & Yield $^{b} / \%$ \\
\hline 1 & NBS (20) & $\mathrm{H}_{2} \mathrm{O} / \mathrm{C}_{2} \mathrm{H}_{5} \mathrm{OH}$ & $1.5 / 0.5$ & 30 & 7 & 77 \\
\hline 2 & NBS (20) & $\mathrm{H}_{2} \mathrm{O} / \mathrm{C}_{2} \mathrm{H}_{5} \mathrm{OH}$ & $1.3 / 0.7$ & 30 & 7 & 81 \\
\hline 3 & NBS (20) & $\mathrm{H}_{2} \mathrm{O} / \mathrm{C}_{2} \mathrm{H}_{5} \mathrm{OH}$ & $1.0 / 1.0$ & 30 & 7 & 69 \\
\hline 4 & NBS (20) & $\mathrm{H}_{2} \mathrm{O} / \mathrm{C}_{2} \mathrm{H}_{5} \mathrm{OH}$ & $0.7 / 0.3$ & 30 & 7 & 88 \\
\hline 5 & NBS (20) & $\mathrm{H}_{2} \mathrm{O} / \mathrm{C}_{2} \mathrm{H}_{5} \mathrm{OH}$ & $1.0 / 0.5$ & 30 & 7 & 90 \\
\hline 6 & NBS (20) & $\mathrm{H}_{2} \mathrm{O}$ & 2 & 30 & 7 & 70 \\
\hline 7 & NBS (20) & $\mathrm{H}_{2} \mathrm{O} / \mathrm{CH}_{3} \mathrm{OH}$ & $1.0 / 0.5$ & 30 & 7 & 85 \\
\hline 8 & NBS (20) & $\mathrm{H}_{2} \mathrm{O} / \mathrm{CH}_{3} \mathrm{COCH}_{3}$ & $1.0 / 0.5$ & 30 & 7 & 76 \\
\hline 9 & NBS (20) & $\mathrm{H}_{2} \mathrm{O} / \mathrm{PEG}-400$ & $1.0 / 0.5$ & 30 & 7 & 82 \\
\hline 10 & Succinimide & $\mathrm{H}_{2} \mathrm{O} / \mathrm{C}_{2} \mathrm{H}_{5} \mathrm{OH}$ & $1.0 / 0.5$ & 30 & 7 & 81 \\
\hline $11^{c}$ & - & $\mathrm{H}_{2} \mathrm{O} / \mathrm{C}_{2} \mathrm{H}_{5} \mathrm{OH}$ & $1.0 / 0.5$ & 30 & 7 & 80 \\
\hline $12^{d}$ & NBS (20) & $\mathrm{H}_{2} \mathrm{O} / \mathrm{C}_{2} \mathrm{H}_{5} \mathrm{OH}$ & $1.0 / 0.5$ & 30 & 7 & 94 \\
\hline $13^{e}$ & NBS (20) & $\mathrm{H}_{2} \mathrm{O} / \mathrm{C}_{2} \mathrm{H}_{5} \mathrm{OH}$ & $1.0 / 0.5$ & 30 & 7 & 90 \\
\hline $14^{d}$ & NBS (15) & $\mathrm{H}_{2} \mathrm{O} / \mathrm{C}_{2} \mathrm{H}_{5} \mathrm{OH}$ & $1.0 / 0.5$ & 30 & 7 & 97 \\
\hline $15^{d}$ & NBS (10) & $\mathrm{H}_{2} \mathrm{O} / \mathrm{C}_{2} \mathrm{H}_{5} \mathrm{OH}$ & $1.0 / 0.5$ & 30 & 7 & 91 \\
\hline $16^{d}$ & NBS (15) & $\mathrm{H}_{2} \mathrm{O} / \mathrm{C}_{2} \mathrm{H}_{5} \mathrm{OH}$ & $1.0 / 0.5$ & 40 & 7 & 97 \\
\hline $17^{d}$ & NBS (15) & $\mathrm{H}_{2} \mathrm{O} / \mathrm{C}_{2} \mathrm{H}_{5} \mathrm{OH}$ & $1.0 / 0.5$ & r.t. & 7 & 92 \\
\hline $18^{d}$ & NBS (15) & $\mathrm{H}_{2} \mathrm{O} / \mathrm{C}_{2} \mathrm{H}_{5} \mathrm{OH}$ & $1.0 / 0.5$ & r.t. & 10 & 97 \\
\hline
\end{tabular}

${ }^{a} 0.5 \mathrm{mmol}$ 水杨醛与 $0.5 \mathrm{mmol}$ 麦氏酸反应. ${ }^{b}$ 分离收率. ${ }^{c}$ 无催化剂. ${ }^{d}$ 麦氏酸 $0.55 \mathrm{mmol} .{ }^{e}$ 水杨醛 $0.55 \mathrm{mmol}$. 
表 2 催化体系的底物适用性扩展 ${ }^{a}$

Table 2 Substrate scope for the synthesis of products 3

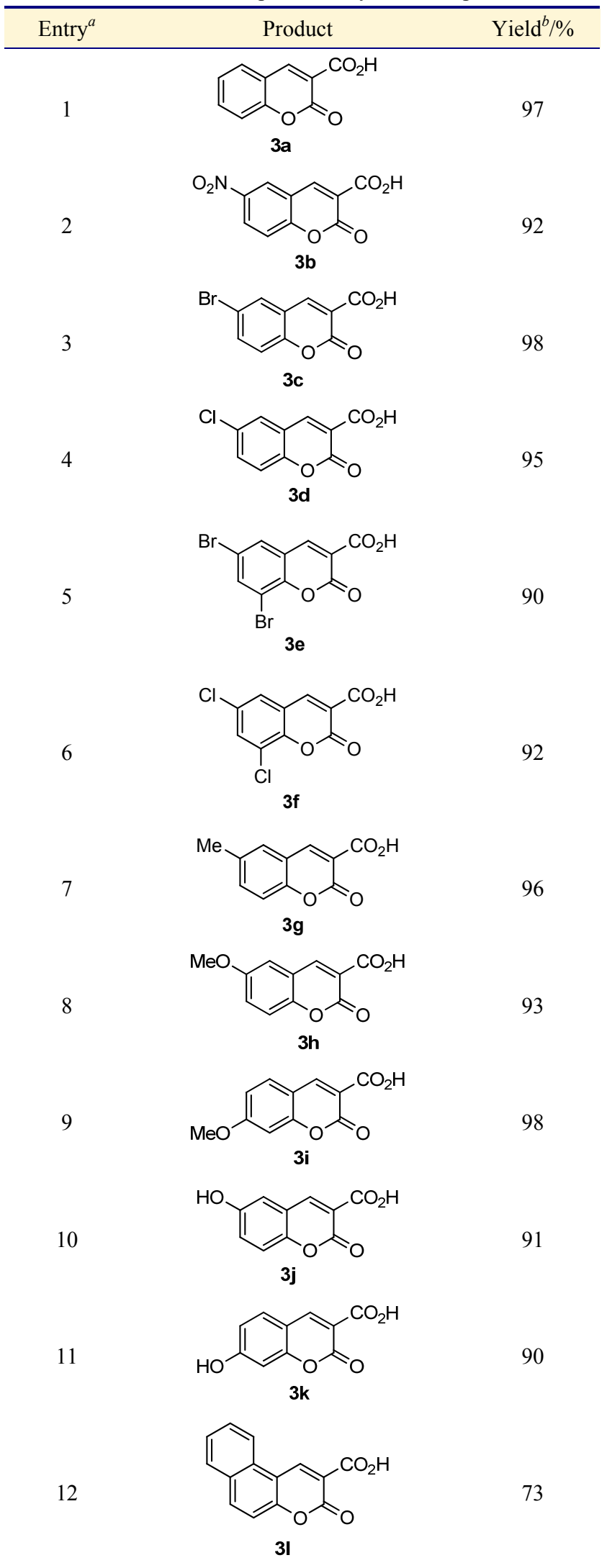

$\overline{{ }^{a}} 0.5 \mathrm{mmol}$ 水杨醛与 $0.55 \mathrm{mmol}$ 麦氏酸室温反应; ${ }^{b}$ 分离收率.
出，该方法的底物适应范围较广，众多取代的水杨醛都 能顺利的进行反应，以较高的收率制备得到目标产物. 含有供电子取代基(表 2, Entries 7 9)和弱拉电子取代 基(表 2, Entries 3,4)的水杨醛醛与未取代的水杨醛获得 的结果相近. 当苯环上连有强拉电子取代基时, 例如硝 基、两个氯原子或澳原子取代的水杨醛(表 2, Entries 2, 5, 6), 对应的产物的产率比未取代的水杨醛略有降低. 羟 基取代的水杨醛稳定性稍差，从而影响反应的收率(表 2, Entries 10,11). 芸基的水杨醛(2-羟基-1-萗甲醛)反应 活性较低, 升高反应温度并延长反应时间后获得中等程 度收率的产物(表 2, Entry 12). 所有的反应结束后都不 需要进行额外的提纯操作, 向反应体系中加入 $0.5 \mathrm{~mL}$ 的 $20 \%$ 的乙醇并在 $100{ }^{\circ} \mathrm{C}$ 下摚拌 $10 \mathrm{~min}$ 就可以达到去 除杂质和催化剂的目的, 充分冷却后抽滤即可获得纯净 的目标化合物, 整个合成和提纯过程简便易行.

根据实验获得的数据和文献报道, 我们推测出可能 的反应机理, 如 Scheme 2 所示. 一方面 NBS 在溶剂中 产生 $\mathrm{Br}^{+}$, 可以活化水杨醛的羰基 ${ }^{[18 ~ 21]}$; 另一方面由于 水的加入可以促进麦氏酸烯醇化, 形成亲核负离子 ${ }^{[45]}$. 经过加成和脱水反应后生成 Knoevenagal 缩合产物. NBS 同样可以活化麦氏酸的羰基，促进分子内环化反 应的进行, 最后经质子转移和丁二酰亚胺负离子的作用 下生成目标产物.

\section{2 结论}

NBS 作为催化剂在水杨醛与麦氏酸的 Knoevenagal 缩合-分子内环化串联反应中表现出较高的活性和较好 的底物适应性. 该策略扩展了可用于合成香豆素衍生物 的催化剂的种类, 与本实验室的前期工作相比, 实现了 在室温条件下合成香豆素-3-羧酸、拓宽了底物的适用范 围、提高了目标产物的产率. 而且该方法不需要大量使 用有机溶剂，不使用金属催化剂，反应结束后不需要复 杂的后处理过程就可以高产率地获得纯净的目标产物, 是香豆素-3-羧酸类化合物的一种简便、有效的合成方 法.

\section{3 实验部分}

\section{1 仪器与试剂}

X-5 型数字熔点仪(温度未经校正); Bruker TENSOR 27 型红外光谱仪, $\mathrm{KBr}$ 压片; Bruker Avance $500 \mathrm{MHz}$ 型 核磁共振仪, $\mathrm{CDCl}_{3}$ 或 DMSO- $d_{6}$ 为溶剂, TMS 为内标. 实验中使用的水杨醛及其它取代的 2-羟基苯甲醛均为 分析纯试剂, 购置于阿拉丁试剂有限公司或萨恩化学技 术有限公司. 丁二酰亚胺购置于萨恩化学技术有限公 司, $N$-澳代丁二酰亚胺购置于阿拉丁试剂有限公司. 


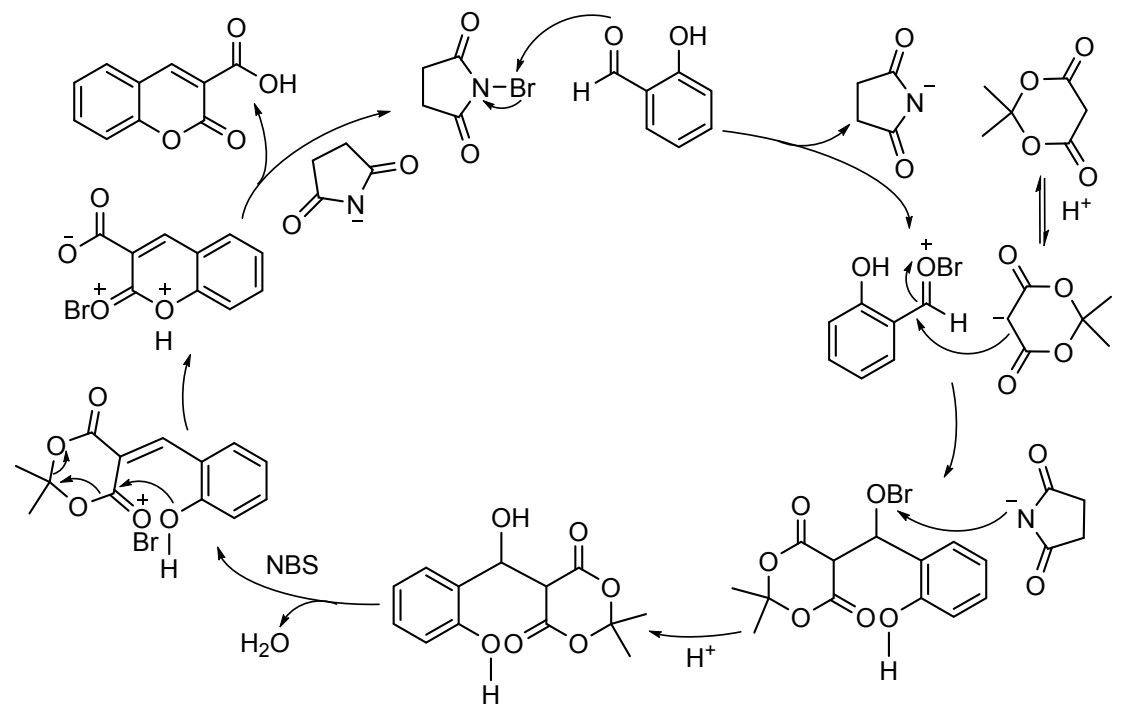

图 2 可能的反应机理

Scheme 2 Possible reaction mechanism

\section{2 目标化合物 $3 a \sim 31$ 的合成}

向 $10 \mathrm{~mL}$ 反应管中加入 $0.5 \mathrm{mmol}$ 水杨醛、 NBS $0.0133 \mathrm{~g}(0.075 \mathrm{mmol}) 、$ 水 $1 \mathrm{~mL}$ 和乙醇 $0.5 \mathrm{~mL}$, 室温摚 拌 3 15 min 后加入麦氏酸 $0.0793 \mathrm{~g}(0.55 \mathrm{mmol})$, 继续 搅拌 $10 \mathrm{~h}$. 反应结束后向体系中加入 $0.5 \mathrm{~mL} 20 \%$ 乙醇, $100{ }^{\circ} \mathrm{C}$ 下摚拌 $10 \mathrm{~min}$, 冷却后抽滤 $\left(3 \mathrm{e}\right.$ 在 $55{ }^{\circ} \mathrm{C}$ 下搅拌 $10 \mathrm{~min}$ ), 用适量冷的 $20 \%$ 乙醇淋洗. 产物不需要再进行 额外的提纯操作就可以得到纯净的化合物, 结构经 IR、 ${ }^{1} \mathrm{H}$ NMR 和 ${ }^{13} \mathrm{C}$ NMR 测定.

香豆素-3-羧酸(3a): 白色固体, m.p. 189 193 ${ }^{\circ} \mathrm{C}$ (文献值 $\left.{ }^{[44]} 188 \sim 190{ }^{\circ} \mathrm{C}\right) ;{ }^{1} \mathrm{H}$ NMR $\left(500 \mathrm{MHz}, \mathrm{CDCl}_{3}\right) \delta$ : $12.18(\mathrm{~s}, 1 \mathrm{H}), 8.96(\mathrm{~s}, 1 \mathrm{H}), 7.81 \sim 7.77(\mathrm{~m}, 2 \mathrm{H}), 7.52 \sim$ $7.48(\mathrm{~m}, 2 \mathrm{H}) ;{ }^{13} \mathrm{C}$ NMR $\left(125 \mathrm{MHz}, \mathrm{CDCl}_{3}\right) \delta: 163.5$, $161.8,154.1,150.9,135.2,130.0,125.7,118.0,116.7$, 114.5; IR (KBr) v: 3062, 1751, 1681, 1612, 1427, 1218, $1041 \mathrm{~cm}^{-1}$.

6-硝基香豆素-3-羧酸(3b): 黄色固体, m.p. 233 $235{ }^{\circ} \mathrm{C}$ (文献值 ${ }^{[4]}$ 231 233 ${ }^{\circ} \mathrm{C}$ ); ${ }^{1} \mathrm{H}$ NMR $(500 \mathrm{MHz}$, $\left.\mathrm{CDCl}_{3}\right) \delta: 9.02(\mathrm{~s}, 1 \mathrm{H}), 8.69(\mathrm{~s}, 1 \mathrm{H}), 8.62(\mathrm{~d}, J=9.1 \mathrm{~Hz}$, $1 \mathrm{H}), 7.66(\mathrm{~d}, J=9.1 \mathrm{~Hz}, 1 \mathrm{H}) ;{ }^{13} \mathrm{C} \mathrm{NMR}\left(125 \mathrm{MHz}, \mathrm{CDCl}_{3}\right)$ $\delta: 161.9,160.4,156.8,149.5,144.6,129.1,125.4,118.1$, 117.8, 116.8; IR (KBr) v: 3286, 3070, 1735, 1612, 1527, $1350,1234,1018,802,740 \mathrm{~cm}^{-1}$.

6-溴香豆素-3-羧酸 (3c): 白色固体, m.p. 194 $196{ }^{\circ} \mathrm{C}$ (文献值 ${ }^{[44]}$ 201 203 ${ }^{\circ} \mathrm{C}$ ); ${ }^{1} \mathrm{H}$ NMR $(500 \mathrm{MHz}$, $\left.\mathrm{CDCl}_{3}\right) \delta: 12.06(\mathrm{~s}, 1 \mathrm{H}), 8.86(\mathrm{~s}, 1 \mathrm{H}), 7.89(\mathrm{~s}, 1 \mathrm{H}), 7.86(\mathrm{~d}$, $J=9.1 \mathrm{~Hz}, 1 \mathrm{H}), 7.39(\mathrm{~d}, J=8.8 \mathrm{~Hz}, 1 \mathrm{H}) ;{ }^{13} \mathrm{C}$ NMR $(125$ $\left.\mathrm{MHz}, \mathrm{CDCl}_{3}\right) \delta: 162.9,161.2,152.8,149.5,137.9,131.9$,
119.3, 118.5, 118.4, 115.5; IR (KBr) v: 3047, 1759, 1674, 1604, 1558, 1365, 1211, 1026, $810 \mathrm{~cm}^{-1}$.

6-氯香豆素-3-羧酸 (3d): 白色固体, m.p. 190 $192{ }^{\circ} \mathrm{C}$ (文献值 ${ }^{[44]} 158 \sim 160{ }^{\circ} \mathrm{C}$ ); ${ }^{1} \mathrm{H}$ NMR $(500 \mathrm{MHz}$, $\left.\mathrm{CDCl}_{3}\right) \delta: 12.08(\mathrm{~s}, 1 \mathrm{H}), 8.88(\mathrm{~s}, 1 \mathrm{H}), 7.73(\mathrm{~d}, J=9.8 \mathrm{~Hz}$, $2 \mathrm{H}), 7.46(\mathrm{~d}, J=8.6 \mathrm{~Hz}, 1 \mathrm{H}) ;{ }^{13} \mathrm{C}$ NMR $\left(125 \mathrm{MHz}, \mathrm{CDCl}_{3}\right)$ $\delta: 162.9,161.2,152.3,149.6,135.0,131.3,128.8,118.9$, 118.1, 115.6; IR (KBr) v: 3042, 1748, 1688, 1555, 1471, 1345, 1248, 1008, 947, $803 \mathrm{~cm}^{-1}$.

6,8-二溴香豆素-3-羧酸 (3e): 淡黄色固体, m.p. $223 \sim 225{ }^{\circ} \mathrm{C}$ (文献值 ${ }^{[44]} 217 \sim 219{ }^{\circ} \mathrm{C}$ ); ${ }^{1} \mathrm{H}$ NMR $(500$ $\left.\mathrm{MHz}, \mathrm{CDCl}_{3}\right) \delta: 11.86(\mathrm{~s}, 1 \mathrm{H}), 8.82(\mathrm{~s}, 1 \mathrm{H}), 8.10(\mathrm{~s}, 1 \mathrm{H})$, $7.83(\mathrm{~s}, 1 \mathrm{H}) ;{ }^{13} \mathrm{C}$ NMR $\left(125 \mathrm{MHz}, \mathrm{CDCl}_{3}\right) \delta: 162.0,160.7$, 149.8, 149.2, 140.4, 131.0, 120.0, 118.4, 116.2, 111.4; IR (KBr) v: 3062, 1766, 1612, 1550, 1434, 1234, $987 \mathrm{~cm}^{-1}$.

6,8-二氯香豆素-3-羧酸 (3f): 乳白色固体, m.p. $207 \sim 209{ }^{\circ} \mathrm{C}$ (文献值 ${ }^{[44]} 201 \sim 203{ }^{\circ} \mathrm{C}$ ); ${ }^{1} \mathrm{H}$ NMR $(500$ $\left.\mathrm{MHz}, \mathrm{CDCl}_{3}\right) \delta: 8.86(\mathrm{~s}, 1 \mathrm{H}), 7.81(\mathrm{~s}, 1 \mathrm{H}), 7.67(\mathrm{~s}, 1 \mathrm{H}) ;{ }^{13} \mathrm{C}$ NMR $\left(125 \mathrm{MHz}, \mathrm{CDCl}_{3}\right) \delta: 161.9,160.7,149.3,148.3$, $134.8,131.1,127.3,123.0,119.6,116.3$; IR (KBr) v: 3062, 1751, 1620, 1450, 1386, 1242, 1064, 1010, $956 \mathrm{~cm}^{-1}$.

6-甲基香豆素-3-羧酸 (3g): 白色固体, m.p. 165 $167{ }^{\circ} \mathrm{C}$ (文献值 ${ }^{[44]} 164 \sim 166{ }^{\circ} \mathrm{C}$ ); ${ }^{1} \mathrm{H}$ NMR $(500 \mathrm{MHz}$, $\left.\mathrm{CDCl}_{3}\right) \delta: 12.29(\mathrm{~s}, 1 \mathrm{H}), 8.89(\mathrm{~s}, 1 \mathrm{H}), 7.58(\mathrm{~d}, J=8.4 \mathrm{~Hz}$, $1 \mathrm{H}), 7.52(\mathrm{~s}, 1 \mathrm{H}), 7.38(\mathrm{~d}, J=8.5 \mathrm{~Hz}, 1 \mathrm{H}), 2.48(\mathrm{~s}, 3 \mathrm{H}) ;{ }^{13} \mathrm{C}$ NMR $\left(125 \mathrm{MHz}, \mathrm{CDCl}_{3}\right) \delta: 163.8,162.0,152.3,150.9$, 136.4, 135.8, 129.5, 117.8, 116.4, 114.2, 20.2; IR (KBr) $v$ : 3062, 1743, 1674, 1604, 1573, 1373, 1218, 1157, 1026, $817 \mathrm{~cm}^{-1}$. 
6-甲氧基香豆素-3-羧酸(3h): 黄色固体, m.p. 206 $208{ }^{\circ} \mathrm{C}$ (文献值 $\left.{ }^{[41]} 192 \sim 194{ }^{\circ} \mathrm{C}\right) ;{ }^{1} \mathrm{H}$ NMR $(500 \mathrm{MHz}$, $\left.\mathrm{CDCl}_{3}\right) \delta: 12.34(\mathrm{~s}, 1 \mathrm{H}), 8.90(\mathrm{~s}, 1 \mathrm{H}), 7.42(\mathrm{~d}, J=9.1 \mathrm{~Hz}$, $1 \mathrm{H}), 7.36(\mathrm{~d}, J=9.2 \mathrm{~Hz}, 1 \mathrm{H}), 7.13(\mathrm{~s}, 1 \mathrm{H}), 3.91(\mathrm{~s}, 3 \mathrm{H}) ;{ }^{13} \mathrm{C}$ NMR $\left(125 \mathrm{MHz}, \mathrm{CDCl}_{3}\right) \delta: 163.6,162.0,156.7,150.6$, $148.7,123.9,118.4,117.8,114.5,110.5,55.5$; IR (KBr) $v$ : 3055, 1759, 1674, 1573, 1496, 1404, 1234, 1026, 802 $\mathrm{cm}^{-1}$.

7-甲氧基香豆素-3-羧酸(3i): 黄色固体, m.p. 192 $194{ }^{\circ} \mathrm{C}$ (文献值 $\left.{ }^{[44]} 194 \sim 196{ }^{\circ} \mathrm{C}\right) ;{ }^{1} \mathrm{H}$ NMR $(500 \mathrm{MHz}$, DMSO- $\left.d_{6}\right) \delta: 12.93(\mathrm{~s}, 1 \mathrm{H}), 8.71(\mathrm{~s}, 1 \mathrm{H}), 7.82(\mathrm{~d}, J=8.6$ $\mathrm{Hz}, 1 \mathrm{H}), 7.02$ (s, 1H), 7.00 (d, $J=8.8 \mathrm{~Hz}, 1 \mathrm{H}), 3.88(\mathrm{~s}, 3 \mathrm{H})$; ${ }^{13} \mathrm{C}$ NMR (125 MHz, DMSO- $\left.d_{6}\right) \delta: 164.6,164.1,157.2$, $156.8,149.0,131.5,113.8,113.2,111.6,100.2,56.2$; IR (KBr) $v: 3047,1728,1612,1504,1380,1218,1018,810$ $\mathrm{cm}^{-1}$.

6-羟基香豆素-3-羧酸 $(3 \mathbf{j})$ : 黄色固体, m.p. > $300{ }^{\circ} \mathrm{C}$ (文献值 ${ }^{[31]} 200{ }^{\circ} \mathrm{C}$ ); ${ }^{1} \mathrm{H}$ NMR (500 MHz, DMSO-d $\left.d_{6}\right) \delta: 13.11(\mathrm{~s}, 1 \mathrm{H}), 9.84(\mathrm{~s}, 1 \mathrm{H}), 8.64(\mathrm{~s}, 1 \mathrm{H}), 7.28$ $(\mathrm{d}, J=8.8 \mathrm{~Hz}, 1 \mathrm{H}), 7.19$ (s, 1H), 7.14 (dd, $J=8.8,1.9 \mathrm{~Hz}$, $1 \mathrm{H}) ;{ }^{13} \mathrm{C}$ NMR $\left(125 \mathrm{MHz}, \mathrm{DMSO}-d_{6}\right) \delta: 164.1,157.2$, $153.9,148.1,147.8,122.4,118.5,118.3,117.0,113.7$; IR (KBr) v: 3417, 3178, 1728, 1573, 1496, 1380, 1249, 1049, $825 \mathrm{~cm}^{-1}$.

7-羟基香豆素-3-羧酸(3k): 黄色固体, m.p. 277 $279{ }^{\circ} \mathrm{C}$ (文献值 ${ }^{[44]} 268 \sim 270{ }^{\circ} \mathrm{C}$ ); ${ }^{1} \mathrm{H}$ NMR $(500 \mathrm{MHz}$, DMSO- $\left.d_{6}\right) \delta: 12.80(\mathrm{~s}, 1 \mathrm{H}), 11.07(\mathrm{~s}, 1 \mathrm{H}), 8.67(\mathrm{~s}, 1 \mathrm{H})$, $7.73(\mathrm{~d}, J=8.6 \mathrm{~Hz}, 1 \mathrm{H}), 6.84(\mathrm{dd}, J=8.6,2.0 \mathrm{~Hz}, 1 \mathrm{H})$, $6.73(\mathrm{~d}, J=1.9 \mathrm{~Hz}, 1 \mathrm{H}) ;{ }^{13} \mathrm{C}$ NMR $\left(125 \mathrm{MHz}, \mathrm{DMSO}-d_{6}\right)$ $\delta: 164.2,163.9,157.6,156.9,149.4,131.9,113.9,112.4$, 110.6, 101.8; IR (KBr) v: 3139, 1712, 1615, 1559, 1490, $1371,1260,1218,1120,1056,910,856 \mathrm{~cm}^{-1}$.

3-氧代-3H-苯并吡喃-2-羧酸(3I): 浅绿色固体, m.p. $240 \sim 242{ }^{\circ} \mathrm{C}$ (文献值 ${ }^{[44]} 235 \sim 237{ }^{\circ} \mathrm{C}$ ); ${ }^{1} \mathrm{H}$ NMR (500 $\left.\mathrm{MHz}, \mathrm{CDCl}_{3}\right) \delta: 12.31(\mathrm{~s}, 1 \mathrm{H}), 9.71(\mathrm{~s}, 1 \mathrm{H}), 8.45(\mathrm{~d}, J=8.4$ $\mathrm{Hz}, 1 \mathrm{H}), 8.25$ (d, $J=9.0 \mathrm{~Hz}, 1 \mathrm{H}), 8.00(\mathrm{~d}, J=8.1 \mathrm{~Hz}, 1 \mathrm{H})$, 7.84 (t, $J=7.7 \mathrm{~Hz}, 1 \mathrm{H}), 7.71$ (t, $J=7.5 \mathrm{~Hz}, 1 \mathrm{H}), 7.59$ (d, $J=9.1 \mathrm{~Hz}, 1 \mathrm{H}) ;{ }^{13} \mathrm{C}$ NMR (125 MHz, DMSO- $\left.d_{6}\right) \delta: 164.2$, $156.7,154.9,143.6,135.7,129.7,128.9,128.8,126.3$, 122.2, 117.0, 116.4, 111.9; IR (KBr) v: 3062, 1743, 1697, $1566,1504,1326,1226,1026,956 \mathrm{~cm}^{-1}$.

辅助材料(Supporting Information) 化合物 3a $\sim 31$ 的 核磁谱图. 这些材料可以免费从本刊网站(http://siocjournal.cn/)上下载.

\section{Referenes}

[1] Safaei, H. R.; Safaei, M.; Shekouhy, M. RSC Adv. 2015, 5, 6797.

[2] Dekamin, M. G.; Eslami, M. Green Chem. 2014, 16, 4914.

[3] Xiong, X.; Han, Q.; Shi, L.; Xiao, S.; Bi, C. Chin. J. Org. Chem. 2016, 36, 480 (in Chinese).

(熊兴泉, 韩寒, 石霖, 肖上运, 毕成, 有机化学, 2016, 36, 480.)

[4] Wei, L.; Chen, X.; Liu, Y.; Wan, J. Chin. J. Org. Chem. 2016, 36, 954 (in Chinese). (韦丽, 陈绪文, 刘云云, 万结平, 有机化学, 2016, 36, 954.)

[5] Zhang, Q.; Vigier, K. D. O.; Royer, S.; Jérôme, F. Chem. Soc. Rev. 2012, 41, 7108 .

[6] Patil, D.; Chandam, D.; Mulik, A.; Patil, P.; Jagadale, S.; Kant, R.; Gupta, V.; Deshmukh, M. Catal. Lett. 2014, 144, 949.

[7] Brahmachari, G.; Banerjee, B. ACS Sustainable Chem. Eng. 2014 $2,411$.

[8] Chandam, D. R.; Mulik, A. G.; Patil, P. P.; Jagdale, S. D.; Patil, D. R.; Deshmukh, M. B. Res. Chem. Intermed. 2015, 41, 761.

[9] Shabalala, N.; Maddila, S.; Jonnalagadda, S. B. New J. Chem. 2016 40, 5107.

[10] Adib, M.; Yasaei, Z.; Mirzaei, P. Synlett 2016, 27, 383.

[11] Venkatesan, K.; Pujari, S. S.; Srinivasan, K. V. Synth. Commun. 2009, 39, 228.

[12] Ghosh, S.; Das, J.; Chattopadhyay, S. Tetrahedron Lett. 2011, 52, 2869.

[13] Sadeh, F. N.; Maghsoodlou, M. T.; Hazeri, N.; Kangani, M. Res. Chem. Intermed. 2015, 41, 5907.

[14] Sui, X. -F.; Yuan, J. -Y.; Zhou, M.; He, Y. -H. Chin. J. Org. Chem. 2006, 26, 1518 (in Chinese). (隋晓锋，袁金颖，周密，何永洪，有机化学, 2006, 26, 1518.)

[15] Xu, D.; Wang, S.; Shen, Z.; Xia, C.; Sun, W. Org. Biomol. Chem. 2012, 10,2730.

[16] Liu, W.; Chen, C.; Qiu, H. Chin. J. Org. Chem. 2015, 35, 450 (in Chinese). (刘卫兵，陈翠，邱会华，有机化学， 2015, 35, 450.)

[17] Saikia, I.; Borah, A. J.; Phukan, P. Chem. Rev. 2016, 116, 6837.

[18] Shinde, M. H.; Kshirsagar, U. A. Green Chem. 2016, 18, 1455.

[19] Talluri, S. K.; Sudalai, A. Org. Lett. 2005, 7, 855.

[20] Ghorbani-Vaghei, R.; Malaekehpoor, S. M. Tetrahedron Lett. 2012 $53,4751$.

[21] Kiyani, H.; Kanaani, A.; Ajloo, D.; Ghorbani, F.; Vakili, M. Res. Chem. Intermed. 2015, 41, 7739.

[22] Xiong, M.; Xi, H.; Fu, Y.; Sun, X. Chin. J. Org. Chem. 2010, 30, 908 (in Chinese) (熊敏秋, 席海涛, 付永胜, 孙小强, 有机化学, 2010, 30, 908.)

[23] Wang, H.; Zheng, W.; Xie F.; Song, H. Chin. J. Org. Chem. 2011, 31,708 (in Chinese) (王辉, 郑伟华, 谢风妮, 宋化灿, 有机化学, 2011, 31, 708.)

[24] Wei, J.; Wang, P.; Jia, Q.; Huang, J.; Du, Z.; Zhang, K.; Wang, J. Eur. J. Org. Chem. 2013, 2013, 4499.

[25] Sandhu, S.; Bansal, Y.; Silakari, O.; Bansal, G. Bioorg. Med. Chem. 2014, 22, 3806.

[26] Niharika, P.; Ramulu, B. V.; Satyanarayana, G. Org. Biomol. Chem. 2014, $12,4347$.

[27] Li, C.; Yang, D.; Yin, B.; Guo, Y. Chin. J. Org. Chem. 2016, 36, 787 (in Chinese). (李长伟，杨栋，尹兵，郭媛，有机化学, 2016, 36, 787.)

[28] He, X.; Yan, Z.; Hu, X.; Zuo, Y.; Jiang, C.; Jin, L.; Shang, Y. Synth. Commun. 2014, 44, 1507.

[29] Rahmani-Nezhad, S.; Khosravani, L.; Saeedi, M.; Divsalar, K.; Firoozpour, L.; Pourshojaei, Y.; Sarrafi, Y.; Nadri, H.; Moradi, A.; Mahdavi, M.; Shafiee, A.; Foroumadi, A. Synth. Commun. 2015, 45,741 .

[30] Vekariya, R. H.; Patel, H. D. Synth. Commun. 2014, 44, 2756.

[31] Undale, K. A.; Gaikwad, D. S.; Shaikh, T. S.; Desai, U. V.; Pore, D. M. Indian J. Chem., Sect. B 2012, 51B, 1039.

[32] Yang, G.; Wang, C.; Fan, S.; Xie, P.; Jin, Q.; Xu, C. Chin. J. Org. Chem. 2015, 35, 1173 (in Chinese). 
(杨国玉，王彩霞，訤素芳，谢普会，金秋，徐翠莲，有机化学， $\mathbf{2 0 1 5}, 35,1173$.

[33] Scott, J. L.; Raston, C. L. Green Chem. 2000, 2, 245.

[34] Alvim, J.; Dias, Jr. , R. L. A.; Castilho, M. S.; Oliva, G.; Corrêa, A. G. J. Braz. Chem. Soc. 2005, 16, 763.

[35] Heravi, M. M.; Sadjadi, S.; Oskooie, H. A.; Shoar, R. H.; Bamoharram, F. F. Catal. Commun. 2008, 9, 470

[36] Li, Y.; Gao, W. Synth. Commun. 2012, 42, 2067.

[37] Karami, B.; Farahi, M.; Khodabakhshi, S. Helv. Chim. Acta 2012, $95,455$.

[38] He, X.; Shang, Y.; Zhou, Y.; Yu, Z.; Han, G.; Jin, W.; Chen, J. Tetrahedron $\mathbf{2 0 1 5}, 71,863$.

[39] Fiorito, S.; Genovese, S.; Taddeo, V. A.; Epifano, F. Tetrahedron
Lett. 2015, 56, 2434

[40] Brahmachari, G. ACS Sustainable Chem. Eng. 2015, 3, 2350.

[41] Chavan, H. V.; Bandgar, B. P. ACS Sustainable Chem. Eng. 2013, 1 , 929.

[42] Fiorito, S.; Taddeo, V. A.; Genovese, S.; Epifano, F. Tetrahedron Lett. 2016, 57, 4795.

[43] Pan, W. -Y.; Xiao, Y. -M.; Xiong, H. -Q.; Lü, C. -W. Res. Chem. Intermed. 2016, 42, 7057.

[44] Gao, S.; Xiao, D.; Yang, Y.; Wei, X.; Sun, S.; Lang, J.; Lv, C. Heterocycles 2016, 92, 1698.

[45] Bigi, F.; Carloni, S.; Ferrari, L.; Maggi, R.; Mazzacani, A.; Sartori, G. Tetrahedron Lett. 2001, 42, 5203.

(Li, L.; Lu, Z.) 